\title{
1. Zur Untersuchung von Raum- und Identitätskonstruktionen in Grenzregionen
}

Christian Wille und Rachel Reckinger

In diesem Band werden Räume und Identitäten in Grenzregionen untersucht. Das so pointiert formulierte Programm beruht auf einer vielschichtigen Forschungskonzeption, die Ansätze der Raum- und Identitätsforschung miteinander verknüpft und verschiedene thematische Zugänge integriert. Den Ausgangspunkt bildet die Auffassung, dass Räume und Identitäten aus sozialen Praktiken hervorgehen. Entsprechende praxistheoretische Ansätze, die verkürzt auch als Doing Space oder Doing Identity wiedergegeben werden können, fokussieren auf den Aufführungs- bzw. Vollzugscharakter, was mit Konzepten wie >Geographie-Machen<, >Ent-/Verräumlichung< oder >Identitätsarbeit< und >Identitätspolitik anschaulich zum Ausdruck kommt. Diese auch hier eingenommene Perspektive bietet nicht nur vielfältige Anknüpfungspunkte für die an diesem Band beteiligten Disziplinen, sie wird auch geradezu herausgefordert bei der Untersuchung von und in Grenzregionen. Denn erst konstruktivistische und kontingenzorientierte $\mathrm{Zu}$ gänge erlauben hier adäquate Zugriffe auf Raum- und Identitätskonstruktionen, schreiben sie sich in Grenzregionen - so die These - doch weniger in >nationalstaatliche Ordnungen < oder in >binäre Ordnungen< des Hier/Dort ein. Vielmehr ist für Grenzregionen von raum- und identitätsbezogenen >Logiken der Unordnung< auszugehen, die sich in Ambivalenzen sowie in >quer liegenden< Artikulationsformen äußern, die jeweils selbst als Grenzregionen oder Zwischenräume qualifiziert werden können, und die Praktiken bewirken, die auf die (Wieder-)Einsetzung von >Ordnungen< abzielen. Diese und weitere Prozesse der Raum- und Identitätskonstruktion sind Gegenstand dieses Bandes, die über institutionelle, mediale und alltagskulturelle Praktiken in Grenzregionen rekonstruiert werden.

Mit diesem Themenaufriss ist bereits angedeutet, dass der Begriff der Grenzregion - und damit der Grenze - in diesem Band unterschiedliche Konkretisierungen erfährt. Zunächst in einem politisch-administrativen Sinn bilden Luxemburg und die angrenzenden Gebiete in Deutschland, Frankreich und Belgien den empirischen Untersuchungskontext der einzelnen Buchbeiträge. Daneben wird der Begriff in einem kategorialen Sinn verwendet, wenn (zumeist dichotom verfasste) 
Ordnungskategorien eingesetzt oder in Frage gestellt werden. Schließlich werden mit dem Begriff >Räume der Grenze < bzw. (kategoriale) Zwischenräume bezeichnet, die über dynamische Differenzverhandlungen hervorgebracht werden.

Für den Begriff der Grenzregion bzw. des Grenzraums ${ }^{1}$ konstitutiv sind also Grenzen bzw. Differenzen, die nicht als fixe und unhinterfragte Setzungen, sondern als Ergebnisse kontingenter Praktiken verstanden werden. Analytisch unterschieden wird dabei zwischen drei miteinander verschränkten >Praktiken der Grenze<, über die sich Räume und Identitäten nicht nur materialisieren, sondern die diese auch mitgestalten: (1) die Einsetzung von Grenzen als Differenzierung bzw. Selbst-/Fremdregulativ zum Außen; (2) die Überschreitung von Grenzen als affirmativer und/oder subversiver Akt mit Transformationspotential und (3) die Ausdehnung von Grenzen als ein >Dazwischen< vielfältiger Relationen und Schnittmengen (vgl. Abschnitt 2.1). Dieses Auffächern von >Praktiken der Grenze $<$ hilft die für Raum- und Identitätskonstruktionen konstitutiven Prozesse der Grenz- bzw. Differenzverhandlung analytisch in den Blick zu nehmen. Dabei geht es in den Fallstudien in diesem Band um praktische Relationierungen bzw. Topologien sowie um die auf die physisch-materielle Welt bezogenen Sinnzuschreibungen, die wiederum Aufschluss geben über Identitätskonstruktionen. Denn während Unterscheidungen, Relationen, das >Sprechen « von einem Hier/ Dort (raumbezogene) Differenzsetzungen anzeigen, geben sie gleichermaßen Auskunft über (Selbst-)Positionierungen und damit über Identitäten.

Diese Annäherung an Raum- und Identitätskonstruktionen - ausgehend und entlang von Grenzeinsetzungen, -überschreitungen und -ausdehnungen - wird in diesem Band konzeptionell weiter ausdifferenziert, so dass zunächst zwischen zwei Untersuchungsperspektiven unterschieden werden kann: Mit Blick auf Raumkonstruktionen geht es um institutionell-mediale Semantisierungen und performative Techniken der Zuweisung und Repräsentation, ebenso wie um alltägliche Geographien als topologische Strukturen und symbolische Verräumlichungen auf Subjektebene (vgl. Abschnitt 2.2). Analog dazu werden Identitätskonstruktionen als Identifikationen und Identifizierungen untersucht, womit einerseits Zuschreibungsprozesse durch bestimmte Instanzen und andererseits alltagskulturelle Aneignungsprozesse (von Zuschreibungen) ins Blickfeld geraten (vgl. Abschnitt 2.3). Beide Untersuchungsperspektiven - die der zugeschriebenen bzw. angeeigneten Räume und Identitäten - bleiben jedoch nicht unverbunden, sondern weiter werden ihre empirischen Verschneidungen und Verknüpfungen in grenzüberschreitenden Bezügen aufgedeckt. Dafür wird v.a. an den Foucaultschen Gouvernementalitätsbegriff angeknüpft - ein Konzept, das sowohl für die Konstruiertheit sozialer Wirklichkeit, für raum- und identitätstheoretische Fra-

1 | Die synonyme Begriffsverwendung von ,Grenzregion ، und 'Grenzraum ‘ in diesem Band ist auf die unterschiedlichen Untersuchungsebenen zurückzuführen und an den Ansatz der "Sozialgeographie alltäglicher Regionalisierungen" (Werlen 1997) rückzubinden (vgl. Abschnitt 2.2). 
gestellungen als auch für das Zusammenwirken unterschiedlicher Aspekte und Ebenen des Sozialen sensibel ist (vgl. Abschnitt 2.3).

Diese drei skizzierten Untersuchungsperspektiven werden in diesem Band im Rahmen von drei Forschungsschwerpunkten bearbeitet. Diese umfassen (1) eine machtkritische Perspektive auf Räume und Identitäten, die sich besonders Politiken und Normierungen zuwendet, die in Konstruktionsprozessen wirksam und verhandelt werden; (2) eine an Medien orientierte Perspektive auf Räume und Identitäten, die Medien als Konstrukteure und Projektionsflächen sowie selbst als (Verhandlungs-)Räume versteht und (3) eine subjektzentrierte Perspektive, die das Hervorbringen von Raum- und Identitätskonstruktionen im Zuge alltagskultureller Praktiken untersucht. Die innerhalb der Forschungsschwerpunke eingenommenen Perspektiven auf Räume und Identitäten ergänzen einander und werden in den Kapiteln 3, 4 und 5 sowohl theoretisch-konzeptionell als auch empirisch über verschiedene Fallstudien entwickelt.

\section{Zur Entstehung dieses Bands}

Der vorliegende Band ist im Rahmen eines Forschungsprojekts an der Universität Luxemburg entstanden. Das von der Universität geförderte Projekt mit dem Titel IDENT2 - Regionalisierungen als Identitätskonstruktionen in Grenzräumen ${ }^{2}$ (20112014) hatte nicht nur ein vielschichtiges Thema zum Gegenstand, ebenso waren daran zahlreiche Wissenschaftler/-innen der Forschungseinheit IPSE - Identités, Politiques, Sociétés, Espaces beteiligt, d.h. ca. 30 Kolleg/-innen der acht Mitgliedsinstitute $^{3}$. Der disziplinenübergreifende Forschungszusammenhang wurde im Anschluss an das Vorgängerprojekt IDENT - Soziokulturelle Identitäten und Identitätspolitiken in Luxemburg (2007-2010) (vgl. IPSE 2010; IPSE 2011a; IPSE 2011b) konzipiert, in dem bereits Identitätskonstruktionen im Zentrum standen. Die dort erzielten Ergebnisse wurden nicht nur fortgeschrieben, sondern im vorliegenden Band spürbar weiterentwickelt. Dies zeigt sich in der erweiterten Fragestellung nach Raumkonstruktion und nach den Besonderheiten grenzüberschreitender Untersuchungskontexte; ebenso wie auf konzeptioneller und struktureller Ebene eine Entwicklung auszumachen ist, was die komplexe Forschungskonzeption und gesteigerte Zusammenarbeit der beteiligten Disziplinen deutlich machen.

2 | Leitung: Prof. Dr. Sonja Kmec und Prof. Dr. Markus Hesse; Koordination: Dr. Rachel Reckinger und Dr. Christian Wille.

3 | Dazu zählen das Institut für Geschlechterforschung, Diversität und Migration, Institut für Geographie und Raumplanung, Institut für Philosophie, Institut für Geschichte, Institut für deutsche Sprache, Literatur und für Interkulturalität, Institut für Romanistik, Medienund Kunstwissenschaften, Institut für luxemburgische Sprache und Literaturen und das Institut für Politikwissenschaft.

4 | Leitung: Prof. Dr. Christian Schulz; Koordination: Dr. Rachel Reckinger und Dr. Christian Wille. 


\begin{tabular}{|l|c|c|c|}
\hline & AG Politiken & AG Medien & AG Subjekte \\
\hline Anzahl der Mitglieder & 6 & 12 & 13 \\
\hline $\begin{array}{l}\text { Anzahl der beteiligten } \\
\text { IPSE-Institute }\end{array}$ & 4 & 6 & 5 \\
\hline
\end{tabular}

Tabelle 1: Zusammensetzung der inhaltichen Arbeitsgruppen im Projekt IDENT2 Regionalisierungen als Identitätskonstruktionen in Grenzräumen

Die Arbeiten zu diesem Band wurden von den beteiligten Wissenschaftler/-innen im Rahmen von thematischen und begleitenden Arbeitsgruppen realisiert. Dabei behandelten die thematischen Arbeitsgruppen jeweils einen der drei Forschungsschwerpunkte, die sie in theoretisch-konzeptioneller und empirischer Hinsicht an der übergreifenden Forschungskonzeption des Projekts ausrichteten. Die Ergebnisse der Zusammenarbeit sind, wie die Kapitel 3, 4 und 5 zeigen, trotz der jeweils individuell bearbeiteten Fallstudien das Resultat regelmäßigen Austauschs und enger Abstimmung. Die inhaltliche Projektarbeit wurde von drei begleitenden Arbeitsgruppen flankiert, die sich auf theoretische Fragen, auf methodische Aspekte und auf die Zusammenarbeit der beteiligten Disziplinen konzentrierten. Die hier geleisteten Arbeiten waren, wie in Kapitel 2 deutlich wird, für die übergreifende Forschungskonzeption und für die Zusammenarbeit der beteiligten Wissenschaftler/-innen von Bedeutung. Der erforderliche Austausch zwischen den inhaltlichen und begleitenden Arbeitsgruppen wurde über die Kolleg/-innen und die Projektkoordination sichergestellt, die in beiden Typen von Arbeitsgruppen vertreten waren.

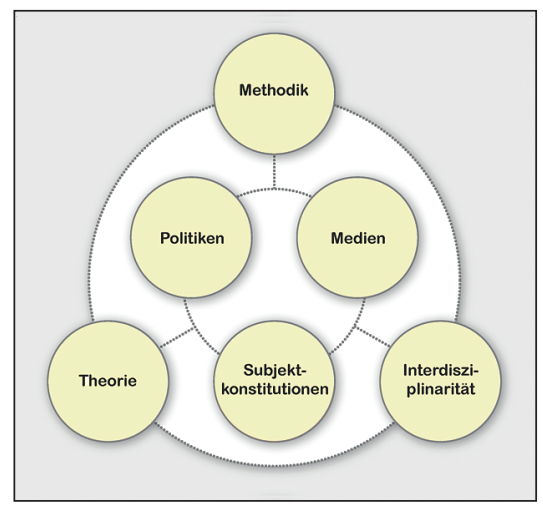

Abbildung 1: Inhaltliche und begleitende Arbeitsgruppen im Projekt IDENT2 Regionalisierungen als Identitätskonstruktionen in Grenzräumen (Entwurf: Christian Wille, Umsetzung: Gilles Caspar und Malte Helfer) 
Abschließend gilt unser Dank allen Personen, ohne die das Projekt IDENT2 Regionalisierungen als Identitätskonstruktionen in Grenzräumen nicht möglich gewesen wäre bzw. die es mitgetragen und damit diesen Band unterstützt haben. Dazu zählen die 3.300 Einwohner/-innen Luxemburgs und der angrenzenden Gebiete, die an der quantitativen und qualitativen Befragung teilgenommen haben, und alle, die uns mit Rat und Tat begleitet haben, insbesondere Gilles Caspar, Tilo Felgenhauer, Georg Glasze, Rouven Hehlert, Peggy Jacobs, Fem Alina Kaup, Bertrand Lévy, Guy di Méo, Birgit Neumann, Peter Schmitt-Egner, Verena Schreiber, Benno Werlen, Ruth Zimmerling, Sabine Zinn-Thomas und viele andere, die nicht namentlich genannt werden. Der Universität Luxemburg danken wir für die großzügige finanzielle Förderung des disziplinenübergreifenden Forschungsprojekts. Nicht zuletzt möchten wir uns bedanken beim transcript Verlag für die professionelle Zusammenarbeit, ebenso wie bei dem Übersetzer und Lektor Matthias Müller, der aus einem mehrsprachigen ${ }^{5}$ und von verschiedenen disziplinären sowie akademischen Traditionen geprägten Buchmanuskript eine deutsche und englische Fassung angefertigt hat.

\section{Literatur}

IPSE (2010) (Hg.): Doing Identity in Luxemburg. Subjektive Aneignungen - institutionelle Zuschreibungen - sozio-kulturelle Milieus. Bielefeld: transcript.

IPSE (2011a) (Hg.): Doing Identity in Luxembourg. Subjective Appropriations Institutional Attributions - Socio-Cultural Milieus. Bielefeld: transcript.

IPSE (2011) (Hg.): Construire des identités au Luxembourg. Appropriations subjectives - Projections institutionnelles - Milieux socio-culturels. Paris: Berg international.

Werlen, Benno (1997): Sozialgeographie alltäglicher Regionalisierungen. Band 2: Globalisierung, Region und Regionalisierung, Stuttgart: Franz Steiner.

5 | Die Buchbeiträge konnten von den beteiligen Wissenschaftler/-innen auf Deutsch, Französisch oder Englisch verfasst werden. 
